\title{
T20QoL: an observational multicenter cohort study to evaluate the quality of life in HIV-patients treated with enfuvirtide (ENF, T-20) in combination with an optimized background therapy
}

R Bucciardini'
M Massella'
A Corpolongo'
P Narciso'
V Fragola'
M Mirra'
S Donnini'
O Viganò
S Costarelli ${ }^{4}$
V Tozzi ${ }^{2}$
'Istituto Superiore di Sanità, Rome,
Italy; ${ }^{2}$ IRCCS L. Spallanzani, Rome,
Italy; ${ }^{3}$ Ospedale L. Sacco, Milan, Italy;
${ }^{4}$ Spedali Civili di Brescia, Brescia, Italy

Correspondence: Raffaella Bucciardini Istituto Superiore di Sanità, Rome, Italy Email r.bucciardini@iss.it

\begin{abstract}
Aim: To evaluate the impact of health-related quality of life (HRQoL) enfuvirtide-based (ENF-based) salvage regimens of treatment-experienced HIV patients, in an observational multicenter cohort study.

Methods: HRQoL was measured in a cohort of 16 patients over a 6-month follow-up using 2 instruments: the ISSQoL (Istituto Superiore di Sanità Quality of Life), a recently validated HIV-specific questionnaire; the EQ-5D (EuroQol), a generic widely used instrument. ENF was given at standard dosage along with an optimized background regimen.

Results: Most of HRQoL dimensions showed improvement in ENF-treated patients at the post-baseline time points. Social functioning was the only dimension showing a negative effect. Monthly care costs of antiretroviral drugs for HIV patients taking ENF plus an optimized background regimen were approximately $€ 2,348$ per patient-month (range $€ 382-€ 2,940$ ).

Conclusion: Our results show that the addition of ENF to an optimized background salvageHAART may positively affect HRQoL not only in clinical trials but also in a sample population of patients used in a routine clinical practice.
\end{abstract}

Keywords: enfuvirtide, HRQoL, HIV

\section{Introduction}

Enfuvirtide (ENF) is the first approved HIV-entry inhibitor, available for clinical use, highly effective as part of a regimen for treatment-experienced patients showing extensive multiclass drug resistance. This drug is also the first and only antiretroviral that requires self-administered subcutaneous injections.

Two large multicenter clinical trials (Lalezari et al 2003; Lazzarin et al 2003) demonstrated the efficacy of ENF-containing regimens on immunologic and virologic responses. ENF-treated patients also experienced significant improvements in several dimensions of health-related quality of life (HRQoL).

These latter findings support the idea that patient-reported outcomes (PROs), such as HRQoL, nowadays play a central role in measuring patient's health status. Although the improvement of quality of life is one of the primary goals of antiretroviral therapy (Guidelines 2007), available data on HIV infection mostly refer to medical aspects, only providing an incomplete assessment of the composite effects of treatment. They lack information that comes directly from the patient. Little patient-centered data have been collected up to now, mostly from clinical trials (Cohen et al 2004; Shalit et al 2007).

The principal aim of this study was to provide information on HRQoL in ENF-treated subjects in a routine clinical practice. 


\section{Methods}

\section{Study treatments and patients}

T20QoL was an observational, longitudinal study to assess the impact of ENF on HRQoL in HIV-positive patients. Data on 16 subjects were collected over a 6-month period. Enrolled HIV-infected patients met the following requirements: 18 years of age or older; patients had to be antiretroviral drug-experienced and fusion inhibitor-naïve; an indication for the initiation of therapy including ENF. Exclusion criteria included pregnancy and patients unable to read or complete the questionnaires. ENF was given at standard dosage ( $90 \mathrm{mg}$ subcutaneous injection, twice a day), along with a selected optimized background regimen. Three large Italian clinical centres (IRCCS L. Spallanzani, Ospedale L. Sacco, Spedali Civili di Brescia) participated in the study. Each center taking part in the study obtained an ethics committee approval and participants provided a written informed consent.

\section{Data collection}

Baseline information included demographic data (gender, age, route of transmission, and HIV disease duration). Clinical information included HIV disease status (CDC disease stage), HIV RNA viral load (VL) and CD4+ cell count. HRQoL measurement data, injection site reactions, adverse events, CD4 count, and VL were collected at baseline and 2 follow-up visits at months 3 and 6 .

\section{HRQoL measurement}

To evaluate HRQoL, the EQ-5D (EuroQoL) and the ISSQoL (Istituto Superiore di Sanità Quality of Life) self-administered instruments were adopted.

The ISSQoL is a recently validated HRQoL specific instrument carefully designed for people living with HIV in the HAART era (Bucciardini et al 2006). This instrument includes two sections: HRQoL Core Evaluation Form and Additional Important Areas for HRQoL. The HRQoL Core Evaluation Form includes 9 Core domains: satisfaction with quality of life (SQL), physical well-being (PW), role well-being (RW), depression and anxiety (DA), energy and vitality (EV), health distress (HD), cognitive functioning (CF), social functioning (SF), and sexual life (SL). The Additional Important Areas for HRQoL include 6 domains: social support (SS), interaction with medical staff (MS), treatment impact (TR), body changes (BC), life planning (LP), motherhood/fatherhood (MF). For this study only the Core Evaluation Form was used. The ISSQoL domains were scored on a $0-100$ scale, with 0 indicating 'poorest' health and 100 indicating 'best' health.
The EQ-5D (Brooks 1996) is a widely used generic questionnaire consisting of 5 domains (mobility, self care, usual activities, pain/discomfort and anxiety/depression) and 3 levels with 1 indicating "no problem", 2 indicating "some or moderate problem" and 3 indicating "extreme problems/impossible to do".

\section{Cost of ARV therapy}

In this study, the only direct costs referred to anti-HIV drugs used as part of salvage regimens were considered. Medical costs were quantified considering National Health Service. Cost estimation was based on data provided by hospital pharmacies, also taking into account discounts offered by pharmaceutical companies to the hospitals.

Calculations were performed on daily costs of each drug, on the basis of standard dosages, the number of patient prescribed medication, and the therapy duration in days. Costs obtained were expressed in terms of "patient-month".

\section{Statistical analysis}

Baseline characteristics (demographic, clinical, and HRQoL) were summarized by descriptive statistics. To estimate the magnitude of HRQoL (ISSQoL and EQ-5D) changes over time the "effect size" was used (Cohen 1977; Guyatt et al 1987; Kazis et al 1989). Effect size was calculated as the ratio of the difference between post-baseline and baseline mean scores, divided by baseline standard deviation.

Injection-site reactions were assessed according to an overall grade that was based on the level of pain and discomfort. Baseline mean changes were examined at 3 and 6 months for VL and CD4+ cell counts.

Statistical calculations were performed using SAS statistical package, version 8.2 (SAS Institute, Inc., Cary, North Carolina, USA).

\section{Results}

\section{Baseline characteristics of the study population}

In this study $88 \%$ of the participants were male; $44 \%$ were heterosexual. Mean age was 44 . The subjects were HIVinfected for a mean of 13 years and $44 \%$ of them had a history of an AIDS-defining event. At baseline, mean HIV viral load was $4.0 \log$ and mean CD4 cell count was 190 cells $/ \mu \mathrm{L}$ (Table 1). The optimized background regimen associated with ENF included a mean of 4 antiretroviral drugs (range 2-5).

Mean baseline ISSQoL domains ranged from 45 to 76 . For EQ-5D profile, $60 \%$ and $80 \%$ of the participants reported "no problems", respectively, for mobility and self-care. 
Table I Population characteristics

\begin{tabular}{|c|c|}
\hline & Patients \\
\hline $\mathbf{N}^{\circ}$, randomized & 16 \\
\hline \multicolumn{2}{|l|}{ Gender } \\
\hline Female: n (\%) & $2(12.5)$ \\
\hline Male: n (\%) & $14(87.5)$ \\
\hline \multicolumn{2}{|l|}{ Age (years) } \\
\hline mean $\pm S D(n$, range $)$ & $44.3 \pm 8.0(16,34-63)$ \\
\hline median & 43.0 \\
\hline \multicolumn{2}{|l|}{ Transmission route: $\mathrm{n}(\%)$} \\
\hline Homo/bisexual & $4(25.0)$ \\
\hline IV drug use & $4(25.0)$ \\
\hline Heterosexual & $7(43.8)$ \\
\hline Blood & I ( 6.3) \\
\hline \multicolumn{2}{|l|}{$\mathrm{CD} 4+/ \mathrm{mm}^{3}$} \\
\hline mean $\pm S D(n$, range $)$ & $190 \pm 124(16,6-405)$ \\
\hline median & 158 \\
\hline \multicolumn{2}{|l|}{ HIV-RNA cp/mL $\left(\log _{10}\right)$} \\
\hline mean $\pm S D(n$, range $)$ & $4.0 \pm 1.0(16,1.7-5.5)$ \\
\hline median & 4.2 \\
\hline \multicolumn{2}{|l|}{ Clinical status: n (\%) } \\
\hline CDCA & $2(12.5)$ \\
\hline $\mathrm{CDCB}$ & $7(43.8)$ \\
\hline CDC C & $7(43.8)$ \\
\hline \multicolumn{2}{|c|}{ Time from HIV diagnosis (years) } \\
\hline mean $\pm S D(n$, range $)$ & $13.4 \pm 4.3(|6,7-2|)$ \\
\hline median & 13.0 \\
\hline
\end{tabular}

Approximately half of these (53\%) had "some problems" in performing usual activities and in the pain/discomfort. "Extreme problems" in anxiety/depression dimension were reported in $27 \%$ of the participants.

\section{HRQoL measurement ISSQOL}

Changes over time in ISSQoL dimension scores, presented as mean QoL scores, and as effect sizes after 3 and 6 months of treatment are shown in Table 2 and Table 3.

A positive "small" effect size for physical well-being, role well-being, and energy/vitality both at 3 and 6 months was observed, and for satisfaction with quality of life and health distress after 6 months was observed. A negative "small" effect size for cognitive functioning after 3 months of follow-up was observed.

A "moderate" negative effect size for the social functioning of -0.6 and -0.3 after 3 and 6 months respectively was observed. No significant change during the follow-up for the anxiety/depression and sexual life was reported.
EQ-5D

On average, the EQ-5D profile of the enrolled sample subjects had improved over time (Table 2). The effect sizes were for all dimensions, at the least "small" (0.20-0.40) in both 3 and 6 months; for the Usual Activities the effect size was moderate (0.50) (Table 3$)$.

\section{Injection-site reactions (ISRs)}

After 3 and 6 months of treatment, 50\% and 64\% of participants reported grade 1 pain due to ISRs, respectively.

Grade 1 Erythema was observed in $50 \%$ of participants after 3 months as well as after 6 months; 1 subject reported grade 4 erythema after 3 months and 1 reported grade 2 after 6 months.

In total, after 3 and 6 months, $71 \%$ of participants reported at least one IRS of any grade. After 3 and 6 months $36 \%$ and $50 \%$ of participants, respectively, reported symptoms of pain and erythema (data not shown).

After 6 months of follow-up no adverse events in our group of participants were observed.

\section{Clinical, virological, and immunological follow-up}

Over a 6-month period no change of clinical status was observed (data not shown). During follow-up, an improvement of both immunological and virological parameters was observed (data not shown). The mean increase in CD4+ cell count was $108 \pm 76$ cells $/ \mu \mathrm{L}$ (range, -43 to 223 ) after 3 months, and $114 \pm 101$ cells $/ \mu \mathrm{L}$ (range, -18 to 354 ) after 6 months of therapy. Compared with baseline values, the mean decrease in plasma HIV RNA was $1.5 \pm 1.0 \log _{10}$ copies/mL after 3 months, and $1.4 \pm 1.2 \log _{10}$ copies/mL after 6 months.

\section{Use of resources}

Monthly care costs of antiretroviral drugs for HIV patients taking ENF plus an optimized background regimen was approximately $€ 2,348$ per patient-month (range $€ 382-€ 2,940)$.

\section{Discussion}

In the current study was investigated the HRQoL in subjects receiving ENF-based salvage HAART in an unselected population of heavily pre-treated HIV-infected patients in Italian clinical centres.

We found that using Cohen's guidelines for the magnitude of the effect size, the impact of the ENF on health status was clinically meaningful (at least small) for most of dimensions at 3 and 6 months in both ISSQoL and EQ-5D questionnaires. 
Table 2 Quality of life scores during the follow-up

\begin{tabular}{|c|c|c|c|}
\hline ISSQOL & Baseline & 3 months & 6 months \\
\hline \multicolumn{4}{|l|}{ QoL core evaluation form } \\
\hline Satisfaction with quality of life & $45 \pm 21(14,17-75)$ & $52 \pm 19(11,25-83)$ & $58 \pm 1 \mathrm{I}(\mathrm{II}, 42-75)$ \\
\hline Physical well-being & $63 \pm 30(15,21-100)$ & $75 \pm 22(13,33-100)$ & $80 \pm 16(11,50-100)$ \\
\hline Role well-being & $62 \pm 38(14,0-100)$ & $79 \pm 32(12,0-100)$ & $77 \pm 24(11,38-100)$ \\
\hline Social functioning & $76 \pm 25(14,38-100)$ & $61 \pm 38(12,0-100)$ & $67 \pm 33(11,0-100)$ \\
\hline Anxiety/Depression & $64 \pm 26(15,25-100)$ & $68 \pm 27(|2,2|-100)$ & $68 \pm 23(12,32-96)$ \\
\hline Energy/Vitality & $50 \pm 29(14,13-100)$ & $60 \pm 29(11,6-94)$ & $64 \pm 20(10,38-94)$ \\
\hline Health Distress & $62 \pm 31(15,13-100)$ & $61 \pm 31(12,13-100)$ & $68 \pm 24(12,25-100)$ \\
\hline Cognitive functioning & $66 \pm 24(15,19-100)$ & $63 \pm 32(13,13-100)$ & $69 \pm 26(12,25-100)$ \\
\hline Sexual life & $45 \pm 28(15,0-85)$ & $42 \pm 32(12,0-90)$ & $4 I \pm 29(\mid 2,0-80)$ \\
\hline \multicolumn{4}{|l|}{ EQ-5D dimensions } \\
\hline Mobility: mean \pm SD ( $n$, range) & $1.5 \pm 0.6(15, I-3)$ & $1.2 \pm 0.4(\mid 3, I-2)$ & $\mathrm{I} . \mathrm{I} \pm 0.3(\mathrm{II}, \mathrm{I}-2)$ \\
\hline no problem (\%) & 60 & 85 & 91 \\
\hline some problem (\%) & 33 & 15 & 9 \\
\hline extreme problem (\%) & 7 & - & - \\
\hline Self-care: mean \pm SD ( $n$, range) & $1.2 \pm 0.4(15,-2)$ & $\mathrm{I} . \mathrm{I} \pm 0.3(\mathrm{I}, \mathrm{I}-2)$ & $1.0 \pm 0.0(I I, I-I)$ \\
\hline no problem (\%) & 80 & 92 & 100 \\
\hline some problem (\%) & 20 & 8 & - \\
\hline extreme problem $/ \%)$ & - & - & - \\
\hline Usual activities: mean \pm SD (n, range) & $1.7 \pm 0.6(14, I-3)$ & $\mathrm{I} .3 \pm 0.5(12, \mathrm{I}-2)$ & $\mathrm{I} .3 \pm 0.5(\mathrm{II}, \mathrm{I}-2)$ \\
\hline no problem (\%) & 33 & 67 & 75 \\
\hline some problem (\%) & 53 & 33 & 25 \\
\hline extreme problem (\%) & 7 & - & - \\
\hline Pain/Discomfort: mean \pm SD ( $n$, range) & $1.7 \pm 0.6(15, I-3)$ & $1.4 \pm 0.5(13, I-2)$ & $\mathrm{I} .4 \pm 0.5(\mathrm{II}, \mathrm{I}-2)$ \\
\hline no problem (\%) & 40 & 62 & 64 \\
\hline some problem (\%) & 53 & 38 & 36 \\
\hline extreme problem (\%) & 7 & - & - \\
\hline Anxiety/Depression mean $\pm S D$ ( $n$, range) & $1.9 \pm 0.8(15,1-3)$ & $1.6 \pm 0.7(13,1-3)$ & $1.4 \pm 0.5(12, I-2)$ \\
\hline no problem (\%) & 40 & 46 & 58 \\
\hline some problem (\%) & 33 & 46 & 42 \\
\hline extreme problem (\%) & 27 & 8 & - \\
\hline
\end{tabular}

A significant negative effect was only observed in the social functioning dimension. However, we found significant improvements in CD4+ cell count and reductions in plasma HIV-RNA.

To date, little information exists on the relationships between HRQoL and ENF-based salvage HAART. In a previous study, Cohen et al (2005) assessed the impact of ENF on HRQoL among patients who experienced failure of HAART, enrolled in two phase 3 trials comparing ENF plus background therapy with background therapy alone. Most of HRQoL dimensions (MOS-HIV) showed an improvement in the ENF arm at all post-baseline time points. Social functioning was the only dimension showing a negative effect.
More recently, Shalit et al (2007) examined baseline predictors of life quality improvements in a prospective open-label study of patients treated with ENF in combination with other antiretrovirals. The results showed significant improvements in most of the MOS-HIV domain scores: physical function, mental health, general health perception, pain, energy, quality of life, cognitive function, health distress and health transition. Social functioning and role functioning, were the only domains which did not have any significant improvements.

A decrease in the social functioning score observed in our study is possibly related to the inconvenience mode of ENF administration as well as to ISRs. Both these 
Table 3 Effect size of changes on ISSQoL and EQ-5D dimensions

\begin{tabular}{lll}
\hline ISSQOL & $\begin{array}{l}\text { Effect size } \\
\text { after 3 months } \\
(*)\end{array}$ & $\begin{array}{l}\text { Effect size } \\
\text { after 6 } \\
\text { months (*) }\end{array}$ \\
\hline $\begin{array}{l}\text { QoL core evaluation } \\
\text { form }\end{array}$ & & \\
$\begin{array}{l}\text { Satisfaction with quality } \\
\text { of life }\end{array}$ & $0.1(1.5 / 19.4)$ & $0.4(9.1 / 21.3)$ \\
Physical well-being & $0.2(5.9 / 26.6)$ & $0.3(6.9 / 25.3)$ \\
Role well-being & $0.2(7.3 / 30.7)$ & $0.3(8.0 / 30.8)$ \\
Social functioning & $-0.6(-14.6 / 25.3)$ & $-0.3(-8.0 / 26.2)$ \\
Anxiety/Depression & $0.1(2.1 / 28.8)$ & $0(1.0 / 28.2)$ \\
Energy/Vitality & $0.2(6.8 / 32.3)$ & $0.3(9.4 / 33.0)$ \\
Health distress & $0(0.0 / 34.5)$ & $0.2(5.7 / 33.7)$ \\
Cognitive functioning & $-0.2(-4.8 / 25.4)$ & $0.0(-1.0 / 24.7)$ \\
Sexual life & $-0.1(-1.9 / 28.6)$ & $0.1(-4.0 / 28.0)$ \\
EQ-5D Dimensions & & \\
Mobility & $-0.3(-0.23 / 0.7)$ & $-0.4(-0.27 / 0.7)$ \\
Self-care & $-0.2(-0.07 / 0.4)$ & $-0.3(-0.09 / 0.3)$ \\
Usual Activities & $-0.5(-0.25 / 0.5)$ & $-0.5(-0.27 / 0.5)$ \\
Pain/Discomfort & $-0.3(-0.23 / 0.7)$ & $-0.3(-0.18 / 0.7)$ \\
Anxiety/Depression & $-0.3(-0.23 / 0.9)$ & $-0.4(-0.33 / 0.9)$ \\
\hline () Betw & & \\
\hline
\end{tabular}

(*) Between breaks: difference post baseline and baseline mean/SD.

possibilities were highlighted by Cohen et al (2004) in their study.

For the injection site reaction, observed at 3 and 6 months, our findings are consistent with previous studies, although the percentages reported by Cohen et al and Shalit et al were higher.

In this study, improvements related to mobility and usual activities dimensions were noteworthy results, showing the recovered ability of the subject to act normally during the daily routine.

Further improvements were with the anxiety/depression and the satisfaction with quality of life dimension scores. An explanation of this result could be the participants' awareness of the availability of an effective therapeutic option. This could possibly induce a more positive future outlook, thus minimizing the potential for anxiety and depression and thus improving the quality of life.

Further information on costs of ENF-based regimens was also provided in our study (Hornberger et al 2005, 2006; Sax et al 2005). Our results are consistent with data reported in literature (Shackman et al 2006), in which the cost of ENF-based regimens was US $\$ 3,770(€ 2,421)$ per month. The cost of salvage therapy without ENF of US $\$ 1,970$ $(€ 1,265)$ per month was also reported (Shackman et al 2006). These data suggest that ENF introduction into HAART leads to a significant increase in drug expenditure.

Our study had some limitations. The number of subjects studied was very small and the observed time of follow-up was short. Therefore, our observations should be elaborated in larger and longer clinical-based observational cohorts.

In summary, our results indicate that the addition of ENF to an optimized background salvage-HAART may positively affect HRQoL in a population sample of subjects seen in a routine clinical practice.

\section{Dislosures}

None of the authors has any conflicts of interest to disclose.

\section{References}

Brooks R, the EuroQoL Group. 1996. EuroQoL: the current state of play. Health Policy, 37:53-72.

Bucciardini R, Murri R, Guarinieri M, et al. 2006. ISSQoL: a new questionnaire for evaluating the quality of life of people living with HIV in the HAART era. Qual Life Res, 15:377-90.

Cohen CJ, Clumeck N, Molina JM, et al. 2004. Health-related quality of life with enfuvirtide (ENF; T-20) in combination with an optimized background regimen. J Acquir Immune Defic Syndr, 37:1140-6.

Cohen J. 1977. Statistical power analysis for the behavioral sciences. New York: Academic Press, p. 8.

Guidelines for the use of antiretroviral agents in HIV-infected adults and adolescents [online]. Accessed June 2007. URL: http://www.aidsinfo. nih.gov/

Guyatt G, Walter S, Norman G. 1987. Measuring change over time: assessing the usefulness of evaluative instruments. J Chronic Dis, 40:171.

Hornberger J, Green J, Wintfeld N, et al. 2005. Cost-effectiveness of enfuvirtide for treatment-experienced patients with HIV in Italy. HIV Clin Trials, 6:92-102.

Hornberger J, Kilby JM, Wintfeld N, et al. 2006. Cost-effectiveness of enfuvirtide in HIV therapy for treatment-experienced patients in the United States. AIDS Res Hum Retroviruses, 22:240-7.

Kazis LE, Anderson JJ, Meenan RF. 1989. Effect sizes for interpreting changes in health status. Med Care, 27(Suppl):S178-89.

Lalezari JP, Henry K, O’Hearn M, et al. 2003. for the TORO 1 Study Group. Enfuvirtide, an HIV-1 fusion inhibitor, for drug-Resistant HIV infection in North and South America. N Engl J Med, 348:2175-85.

Lazzarin A, Clotet B, Cooper D, et al. 2003. for the TORO 2 Study Group. Efficacy of enfuvirtide in patients infected with drug-resistant HIV-1 in Europe and Australia. N Engl J Med, 348:2186-95.

Sax PE, Losina E, Weinstein MC, et al. 2005. Cost-effectiveness of enfuvirtide in treatment-experienced patients with advanced HIV disease. J Acquir Immune Defic Syndr, 39:69-77.

Schackman BR, Gebo KA, Walensky RP, et al. 2006. The life time cost of current human immunodeficiency virus care in the United States. Med Care, 44:990-7.

Shalit P, True A, Thommes JA; on behalf of QUALITE' Investigators. 2007. Quality of Life and tolerability after administration of enfuvirtide with a thin-walled needle: QUALITE' Study. HIV Clin Trials, 8:24-35. 
